\title{
p53 gene transfer does not enhance E2F-1-mediated apoptosis in human colon cancer cells
}

\author{
John M. Draus ${ }^{1}$, Mary Jane Elliott ${ }^{1}$, Cesar Atienza, \\ Jr. ${ }^{1}$, Ariel Stilwell ${ }^{1}$, Sandra L. Wong ${ }^{1}$, Yanbin Dong ${ }^{1}$, \\ Hailiang Yang ${ }^{1}$ and Kelly M. McMasters ${ }^{1,2}$
${ }^{1}$ Department of Surgery, University of Louisville, James Graham Brown Cancer Center, Louisville, KY, USA, 40202
${ }^{2}$ Correspondence: Tel, +1-502-852-5211; Fax, +1-502-852-8031; E-mail: kelly.mcmasters@nortonhealthcare.org

Accepted 16 November 2001

Abbreviations: SE, standard error; PBS, phosphate-buffered saline; PARP, poly-ADP ribose polymerase; CDK, cyclin-dependent kinase

\begin{abstract}
E2F-1 and p53 are sequence specific transcription factors that are intimately involved in the regulation of the cell cycle. In addition to their role in cell cycle control, both E2F-1 and p53 have been identified as tumor suppressors and mediators of apoptosis. We have shown previously that adenoviral-mediated E2F-1 overexpression induces efficient apoptosis in colon adenocarcinoma cells. Previous reports have suggested that E2F-1 and p53 cooperate to mediate apoptosis and therefore, in this study, we examined the efficacy of combination gene therapy using adenovirus vectors expressing E2F-1 and p53 in human colon adenocarcinoma cell lines, HT-29 and SW620 (both mutant p53). Cells were treated by mock infection or infection with adenoviral vectors expressing b-galactosidase (LacZ), E2F-1, p53 or a combination of E2F-1 and p53. $I_{25}$ concentrations of each virus were estimated and used for each treatment in order to detect any synergistic or cooperative effects on tumor cell death in the combination therapy. By 5 days post infection, E2F-1-overexpressing cells exhibited growth inhibition and approximately $40-50 \%$ cell death in both cell lines. Co-expression of p53 with E2F-1 abrogated E2F-1-mediated growth inhibition and cell death. Cell cycle analysis revealed that overexpression of E2F-1 resulted in an accumulation of cells in G2/M phase, while overexpression of p53 resulted in a G1 phase accumulation. However, co-expression of E2F-1 and p53 counteracted each other as fewer cells accumulated in G1 and G2/M when compared to either p53 or E2F-1 alone. Furthermore, co-expression of p53 with E2F-1 resulted
\end{abstract}

in decreased levels of E2F-1 protein expression. Mechanistically, upregulation of the CDK inhibitory protein, p21 WAF1/CIP1, was demonstrated in HT-29 cells following overexpression of either E2F-1, p53 or the combination E2F-1/p53 therapy. However, in SW620 cells, only the cells infected with Ad-p53 alone or in combination resulted in upregulation of $\mathrm{p} 21^{\mathrm{WAF} 1 / \mathrm{CIP} 1}$. These results suggest that p53 and p21 ${ }^{\mathrm{WAF} 1 / \mathrm{CIP} 1}$ may cooperate to inhibit the expression and activity of E2F-1. In conclusion, combination adenoviral vector-mediated E2F-1 and p53 gene transfer was not therapeutically advantageous in this in vitro model of human colon adenocarcinoma.

Keywords: E2F-1, p53, p21, gene therapy, apoptosis, adenovirus, colon cancer, cell cycle

\section{Introduction}

E2F-1 is a member of the E2F family of transcription factors that regulates cell cycle progression from $\mathrm{G} 1$ to $S$ phase. E2F-1 forms a heterodimer with a member of the DP family (DP-1 or DP-2) and binds to DNA in a sequence specific manner with high affinity (Dyson, 1998). It has been well established that the gene products of E2F-1 transcriptional activity can promote cells to enter $S$ phase of the cell cycle (Helin, 1998). Although early studies with E2F-1 suggested that it functions as an oncogene (Johnson et al., 1993), recent studies suggest that E2F-1 may also paradoxically function as a tumor suppressor gene, based on its ability to induce apoptosis under conditions of deregulated expression in several normal and malignant cell types (Hunt et al., 1997; Liu et al., 1999; Fueyo et al., 1998). In fact, E2F-1 has been linked to two very distinct apoptotic pathways. First, E2F-1 protein levels increase in a variety of cell lines following DNA damage-induced apoptosis and induction of apoptosis occurs independent of pRb or p53 status (Helin, 1998; Meng et al., 1999). Second, that overexpression of E2F-1 results in p14 ${ }^{\mathrm{ARF}}$-associated apoptosis in both p53-wild-type and p53-null cells. (Bates et al., 1998; Elliott and McMasters, unpublished data).

The p53 tumor suppressor protein is a negative regulator of cell growth. After a cell has incurred DNA damage, the induction of p53 serves either as a key regulator of a G1 cell cycle checkpoint or as an inducer of apoptotic cell death (Ko and Prives, 1996). The expression of p53 following genotoxic stress has long been associated with the transactivation of the p21 WAF1/CIP1 gene (Kastan 
et al., 1991; El-Deiry et al., 1993; Nelson and Kastan, 1994). The $\mathrm{p} 21^{\mathrm{WAF} 1 / \mathrm{CIP} 1}$ protein in turn inhibits the function of specific cyclin-dependent kinases responsible for G1/S transition (El-Deiry et al., 1993; Harper et al., 1993). By inhibiting these kinases, p21 $1^{\text {WAF } 1 / C I P 1}$ induction results in a G1 cell cycle arrest. Alternatively, the stimulation of apoptosis by p53 may result, at least in part, from transcriptional upregulation of the death-promoting gene Bax, which has been shown to contain sequence specific p53-binding sites in its promoter region and from the downregulation of the survival factor Bcl-2 and related Bcl-2 family members (Miyashita et al., 1994; Miyashita and Reed, 1995; Kernohan and Cox, 1996). Importantly, the mechanism by which p53 dictates the choice between $\mathrm{G} 1$ arrest and apoptotic pathways remain ambiguous.

Although E2F-1 is capable of independently inducing apoptosis in certain mammalian cells (Hunt et al., 1997; Dong et al., 1999; Yang et al., 1999), much attention has been given to the role that wild-type p53 may play in that process. Previous studies have shown that E2F1 and p53 cooperate to mediate apoptosis (Qin et al., 1994; Wu and Levine, 1994; Kowalik et al., 1995; Sladek, 1996; Phillips et al., 1997; Hsieh et al., 1997; Kowalik et al., 1998). In some tumor cells, wild-type p53 has been shown to enhance apoptosis with E2F-1 when both were overexpressed. For example, E2F-1-induced apoptosis in fibroblasts is potentiated by high levels of endogenous wild-type p53 (Kowalik et al., 1995; Hsieh et al., 1997). There is also evidence that overexpression of E2F-1 induces the accumulation of p53, again implicating p53 in E2F-1 mediated apoptosis (Hiebert et al., 1995; Kowalik et al., 1998).

We have shown previously that adenoviral-mediated E2F-1 overexpression at high concentrations [multiplicity of infection (MOI) of 100] induces a G2/M arrest and efficient apoptosis in a variety of cancer cell types, including colon adenocarcinoma cells (Dong et al., 1999; and Yang et al., 1999; unpublished data). This study was designed to investigate the potential synergistic effects of expressing E2F-1 and p53 on colon cancer cell death. The results of this study demonstrate that adenovirus-mediated p53 gene transfer is not associated with an increased susceptibility of colon cancer cells to E2F-1-mediated apoptosis. In fact, concomitant adenovirus-mediated overexpression of p53 resulted in a marked inhibition of E2F-1 expression and activity, corresponding with induction of p21 $1^{\mathrm{WAF} 1 / \mathrm{CIP} 1}$. These findings suggest that p53 and p21 may cooperate to inhibit the expression and apoptotic activity of E2F-1.

\section{Materials and Methods}

\section{Cells and culture conditions}

Human colon adenocarcinoma cell lines, HT-29 and
SW620 (both mutant p53), were purchased from ATCC (Rockville, MD). Both cell lines were grown as monolayers in McCoy's 5A medium, supplemented with $10 \%$ heat-inactivated fetal bovine serum and penicillin/ streptomycin (100 units $/ \mathrm{ml})$. The transformed embryonic kidney cell line, 293, was grown in Minimum Essential Media [medium with high glucose $(4.6 \mathrm{~g} / \mathrm{l})$ ], supplemented with $10 \%$ FBS and $1 \%$ antibiotic/antimycotic. Cells were cultured in a $5 \% \mathrm{CO}_{2}$ incubator at $37^{\circ} \mathrm{C}$ and subcultured every 3 days. All cell culture reagents were obtained from Gibco/BRL (Bethesda, MD).

\section{Preparation of recombinant adenovirus}

A replication-defective recombinant adenoviral vector (Ad5 ), with portions of the early region (E1) deleted and a constitutive CMV promoter inserted, was used in these experiments. The adenovirus Ad5CMV-E2F-1, Ad5CMVLacZ, and Ad5CMV-p53 (here in called Ad-E2F-1, AdLacZ and Ad-p53) were generous gifts from Drs. Ta-Jen Liu (M.D. Anderson Cancer Center), Brent French (University of Virginia), and Bert Vogelstein (Johns Hopkins University). For preparation of large virus stocks, 293 cells were infected at MOI 1-5 and harvested after the CPE (cytopathic effect) became visible (24-48 h). Cells were harvested and lysed in $1 \mathrm{X} \mathrm{PBS}^{+2}$ containing $1 \%$ sucrose, and virus aliquots were stored at $-70^{\circ} \mathrm{C}$. Titers were determined by plaque assay on 293 cells. Ad-LacZ (expressing nuclear-localized $\beta$-galactosidase) was used as a control vector and contained no therapeutic gene.

\section{Dose response curves}

HT-29 and SW 620 cell lines were seeded in 6-well plates at $2 \times 10^{5}$ total cells and incubated for $24 \mathrm{~h}$ at $37^{\circ} \mathrm{C}$. One 6-well plate was infected with $200 \mu \mathrm{l}$ of media (mock). Separate 6-well plates were infected with Ad-LacZ, Ad-E2F-1 and Ad-p53 at increasing MOls (10, $25,50,100$, and 150) for a period of $2 \mathrm{~h}$. Four $\mathrm{ml}$ of media supplemented with $5 \%$ FBS and $1 \%$ penicillin/ streptomycin were added to the cells and allowed to incubate for 5 days. Cells were then harvested with trypsin-EDTA. A cell count was obtained with a Coulter counter, and Trypan Blue assays were performed to determine cell viability. Total viable cells were calculated (\% viability $X$ cell count).

\section{Recombinant virus infection}

HT-29 and SW620 cell lines were seeded at $2.5 \times 10^{4}$ cells $/ \mathrm{ml}$ in $75 \mathrm{~mm}$ flasks and allowed to incubate for 24 hours at which time the cells were $35 \%$ confluent. Cells were treated with replication defective recombinant adenovirus vectors for two hours, and then fresh medium was added with $5 \%$ FBS. Both cell lines were treated under five infection conditions as follows: AdE2F-1 infection, Ad-p53 infection, Ad-E2F-1 plus Ad-p53 infection (simultaneous infection), mock infection (control) 
and Ad-LacZ infection (control). Cells were treated with replication defective recombinant adenovirus vectors at $\mathrm{IC}_{25}$ concentrations (Ad-E2F-1 MOI 20 and Ad-p53 MOI 20) for $2 \mathrm{~h}$ and then fresh medium was added. Nonspecific viral toxicity was controlled by using LacZ adenovirus thereby ensuring equivalent viral concentrations. Cells were trypsinized at 24,72 and $120 \mathrm{~h}$ time points, counted via a coulter counter and prepared for various testing as outlined below.

\section{Cell growth and cell viability}

Cell growth (cells $/ \mathrm{ml}$ ) was estimated by counting $100 \mu \mathrm{l}$ of cells with a Coulter Counter at each time point. Cell number provides an estimate of the effect of therapy on the inhibition of cellular proliferation, which includes both cell cycle arrest and cell death. Viability was estimated as the proportion of cells that excluded $0.2 \%$ trypan blue. Cells were stained for $5 \mathrm{~min}$, loaded onto a hemocytometer and scored as either alive or dead using a 10 square count method.

\section{Western blot analysis}

Cells were harvested, centrifuged and washed twice with cold $1 \mathrm{X}$ PBS. The pellets were lysed in lysis buffer (50 mM Tris.Cl, $150 \mathrm{mM} \mathrm{NaCl}, 1 \% N P-40,0.5 \%$ Sodium Deoxycholate and $0.1 \%$ SDS) with a protease inhibitor cocktail [4-(2-aminoethyl)-benzenesulfonyl fluoride (AEBSF), pepstatin $A$, transepoxysuccinyl-L-leucylamido(4-guanidino)butane(E-64), bestatin, leupeptin and aprotinin]. The protein concentration was determined by BIO-RAD protein assay (BIO-RAD, Hercules, CL). For the detection of E2F-1, p53 and actin, $30 \mu \mathrm{g}$ of protein was loaded in separate lanes of a $10 \%$ sodium dodecyl sulfate (SDS)-polyacrylamide gel. A $12 \%$ SDS-polyacrylamide gel was used for the detection of $\mathrm{p} 21$. Proteins were transferred overnight to a polyvinylidone difluoride (PVDF) membrane (BIO-RAD). Proteins were detected using antihuman E2F-1, p53, p21 and actin polyclonal antisera (Sigma). Anti-mouse and anti-rabbit secondary antibodies conjugated to horseradish peroxidase (Amersham) were utilized. Enhanced chemiluminescence $(E C L)$ reagents were used to detect the signals according to the manufacturer's instructions (Amersham, Arlington Heights, IL).

\section{Cell cycle analysis}

For cell cycle analysis, cell suspensions were washed twice with ice-cold PBS, fixed in $70 \%$ cold ethanol and stored at $4^{\circ} \mathrm{C}$ until all time points were collected. Samples were then washed twice with ice-cold PBS and resuspended in $500 \mu \mathrm{l}$ of PBS containing $5 \mu \mathrm{g} / \mathrm{ml}$ propidium iodide and $500 \mu \mathrm{g} / \mathrm{ml}$ RNase A. Following a 20-min incubation at room temperature in the dark, flow cytometric analysis was performed using a FAC-scan (Becton-Dickinson). Data was analyzed using a CellFit Cell-Cycle Analysis program (version 2.01.2). The subdi- ploid population was calculated as an estimate of the apoptotic cell population.

\section{Confirmation of apoptosis}

In addition to DNA fragmentation estimated from the subG1 population, apoptosis was confirmed morphologically by spinning 15,000 cells onto a microscope slide and staining with Wright/Giemsa stains. One thousand cells were counted and scored as either normal, or apoptotic (condensed, intense chromatin staining). In addition, Poly (ADP-ribose) polymerase (PARP) cleavage assays were performed using a monoclonal mouse anti-PARP antibody (Calbiochem, Oncogene Research Products) at a dilution of 1:100.

\section{Statistics}

Unless stated otherwise, numerical data presented are the means of at least two experiments. SEs are shown and Student's t-test was used to evaluate whether differences between data sets were statistically significant. Only P-values $<0.05$ were considered significant.

\section{Results}

\section{Dose response assays}

Cell count and viability data were collected 5 days after infection of HT-29 and SW620 cell lines with increasing amounts of Ad-E2F-1 and Ad-p53 viral vectors. Dosedependent inhibition of total viable cell growth (\% viability $x$ cell count) was demonstrated following infection with both E2F-1 and p53 viral vectors (Figure 1). Transduction with control virus Ad-LacZ did not result in significant nonspecific viral toxicity at an $\mathrm{MOI} \leq 100 . \mathrm{IC}_{25}$ (concentration that inhibited total viable cell growth by $25 \%$ ) values were calculated based on total viable cells and used for all subsequent experiments. Approximate $\mathrm{IC}_{25}$ values were calculated and found to be MOI 20 for Ad-E2F-1 and MOI 20 for Ad-p53. This sub-lethal amount of virus was selected for this study so that possible cooperative effects of simultaneous E2F-1/p53 overexpression could be identified.

\section{E2F-1 and p53 Overexpression}

Western blot analysis of HT-29 and SW620 protein lysates revealed significant baseline expression of the mutant p53 endogenous protein in both cell lines (Figure 2). Mutant p53 in the HT-29 cell line has a lower molecular weight than the adenoviral wild-type p53 but a higher molecular weight than the virally-expressed p53 in SW620 cells. In addition, previous data indicate that the p53 gene mutations found in these two colon cancer cell lines do not result in dominant negative p53 molecules (Goldsmith et al., 1995; Merchant et al., 1996). Baseline expression of exogenous E2F-1 was 

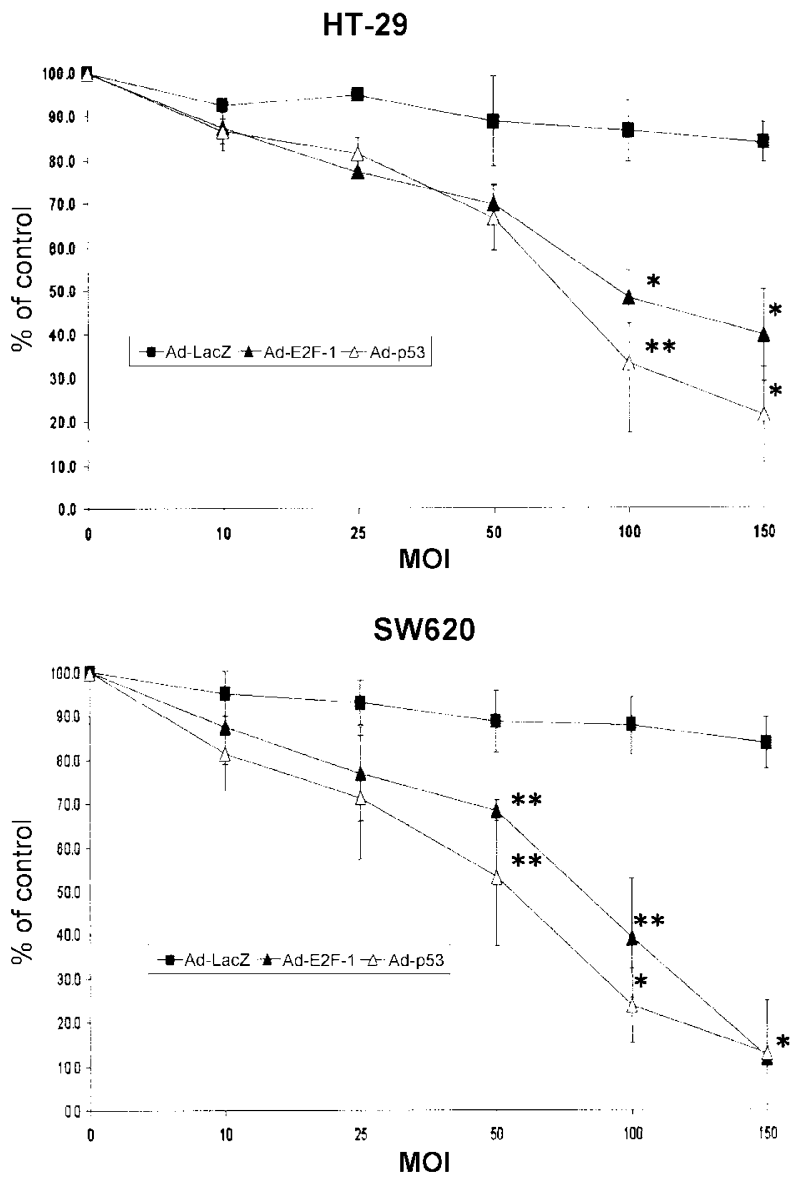

Figure 1. The effect of Ad-E2F-1 and Ad-p53 on viable cell growth of colon cancer cells. Total viable cells were determined by trypan blue exclusion assay and cell counting. Dose response curves demonstrating dosedependent reduction in total viable cells with increasing multiplicity of infection (MOI) from 10 to 150 with Ad-E2F-1 and Ad-p53 and minimal nonspecific viral toxicity with $\mathrm{Ad}-\mathrm{LacZ}$ control at an $\mathrm{MOI}$ of $\leq 150$. Results are expressed as a percent of mock control. Each data point represents the mean $\pm \mathrm{SE}$ of three separate experiments. $P$-values vs Ad-LacZ control are ${ }^{*} \leq .005$ and ${ }^{* *} \leq .05$.

undetectable in control groups treated with mock infection and Ad-LacZ. Substantial overexpression of E2F-1 and p53 was evident after Ad-E2F-1 and Ad-p53 infections, respectively. However, E2F-1 expression was greatly reduced in the E2F-1/p53 combination treatment. Furthermore, both endogenous and viral p53 expression were inhibited following co-infection with the E2F-1 and p53 adenoviruses. These findings suggest negative regulation between E2F-1 and p53 in these colon cancer cells.

\section{Effects on cell growth}

A Coulter Counter ZM was used to perform in vitro growth analysis of our colon cancer cell lines under various infection conditions. After one day, cells infected with Ad-E2F-1, Ad-p53 and Ad-E2F-1/Ad-p53 displayed
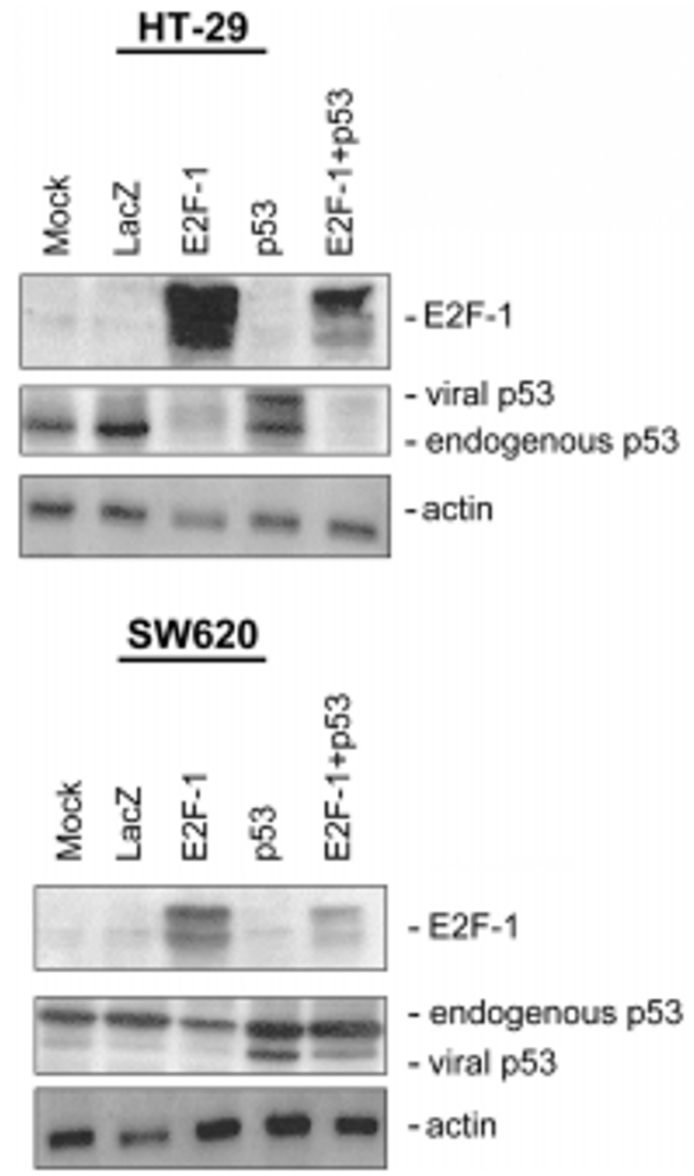

Figure 2. Overexpression of E2F-1 and p53 through adenoviral gene transfer in human colon adenocarcinoma cell lines. The HT-29 and SW620 cell lines were treated by mock infection or infection with adenoviral vectors expressing-galactosidase (LacZ), E2F-1 or p53 in $75 \mathrm{~mm}$ flasks. Blot represents cells harvested at 5 days and lysed in RIPA lysis buffer. Blot is representative of at least three separate experiments. Actin is shown to demonstrate similar levels of protein loading for each of the samples.

no apparent growth inhibition as compared to mockinfected and Ad-LacZ-infected control groups (Figure 3). By three days, cells expressing E2F-1 displayed marked growth inhibition compared to controls. This inhibition became even more pronounced after five days. Cells expressing p53 displayed a similar, although somewhat lower, growth inhibition after three and five days. On day five, cells overexpressing E2F-1 exhibited a 3.8 to 6 fold reduction in cell growth versus control cells, and cell growth was reduced 2.5 to 2.7 fold in the p53-overexpressing cells. Cells co-expressing E2F-1/p53 revealed a 3.0 to 4.3 fold reduction in growth by 5 days post infection when compared to control groups which was between the level of cell growth reduction demonstrated in the cells expressing either E2F-1 or p53 only. The apparent inhibition of growth demonstrated following infection with Ad-E2F-1, Ad-p53 or a combination thereof is most likely due to a combination of cell cycle growth arrest (Figure 5) and cell death (Figure 4). These 
HT-29

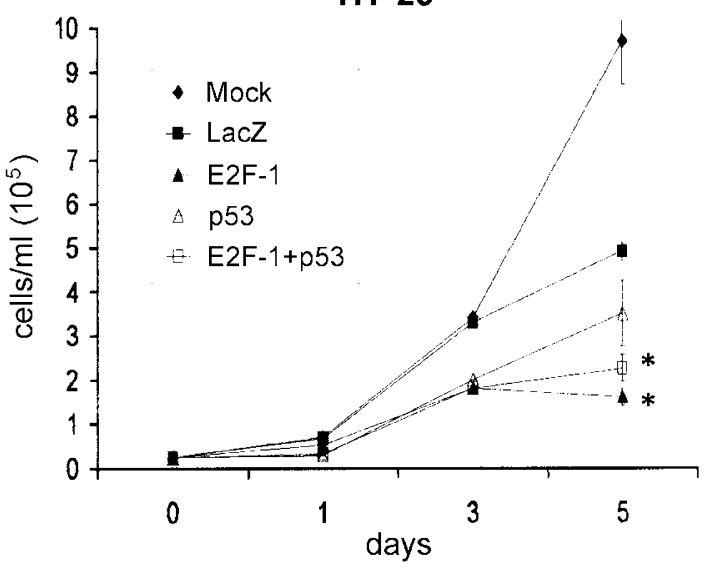

SW620

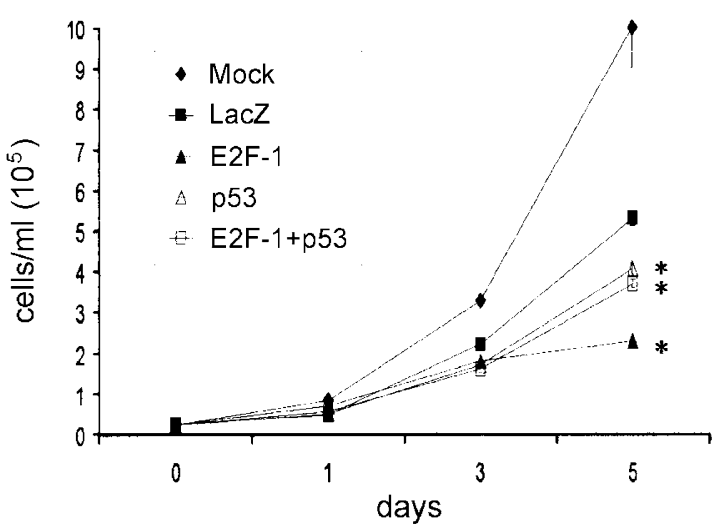

Figure 3. Cell growth analysis of HT-29 and SW620 cells under various gene transfer conditions. Cell growth was determined by counting with a Coulter counter ZM, and results are expressed as cells/ml at time points 1 , 3,5 days post infection. SEs are shown. ${ }^{*} P$-values vs Ad-LacZ control $<0.05$.

findings indicate that E2F-1 and p53 do not cooperate to enhance growth inhibition.

\section{Effects on cell viability}

The effects of E2F-1 and p53 gene overexpression on cell viability in vitro were assessed by trypan blue exclusion. Cells treated with mock infection or Ad-LacZ infection showed little reduction in cell viability after 5 days (Figure 4). Cells infected with Ad-E2F-1, Ad-p53 or Ad-E2F-1/Ad-p53 displayed viability similar to controls after 1 and 3 days. However, by 5 days post infection, only $45.9 \%$ to $53.2 \%$ of the Ad-E2F-1-treated cells were viable, while $75.2 \%$ to $78.0 \%$ of the Ad-p53 treated cells remained viable. The viability of cells co-expressing E2F1 and p53 was $70.7 \%$ and $65.2 \%$. Thus, the combination E2F-1/p53 treatment did not result in a cooperative effect on colon cancer cell death.

\section{Cell cycle analysis}

To further assess the effects of E2F-1 and p53 co-
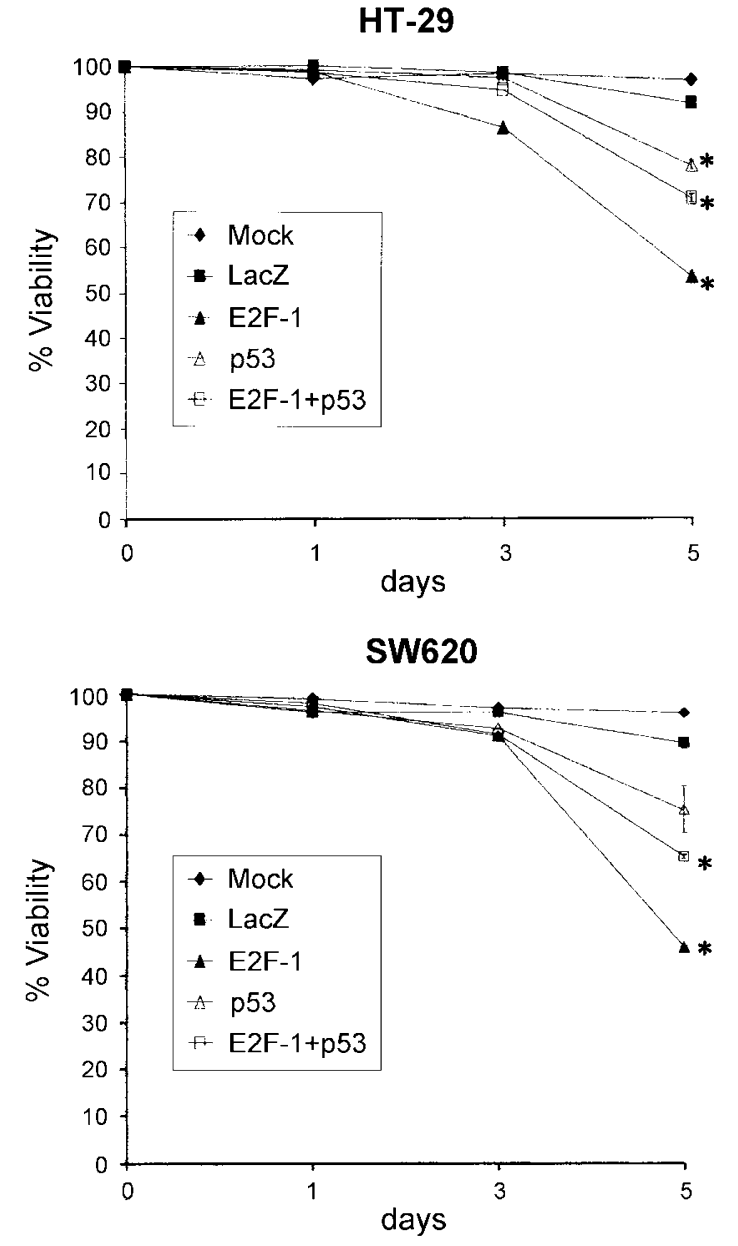

Figure 4. Loss of cell viability after infection with adenoviral vectors expressing E2F-1, p53 or a combination of each. At 1,3 and 5 days after mock infection, or infection with adenovirus expressing LacZ, E2F-1, p53 or combination, both floating and adherent cells were harvested, counted and scored for viability by the trypan blue exclusion method. Results are expressed as the percentage of viable cells (live cells/total cells) mean $\pm \mathrm{SE}$. ${ }^{*} P$-values vs Ad-LacZ control $<0.01$.

expression, cell cycle distribution was analyzed. Infections using $\mathrm{Ad}-\mathrm{E} 2 \mathrm{~F}-1$ alone at an $\mathrm{MOI}$ of 100 was associated with a decrease in $\mathrm{G} 1$ population and a notable increase in the G2/M population of cells (Ad$\mathrm{E} 2 \mathrm{~F}-1=42$ to $60 \%$ vs. controls $=25-33 \%$ ) by one day following treatment (data not shown). Ad-E2F-1, at the reduced $\mathrm{MOI}$ of 20 , still caused a reduction in G1 populations and an increase in the G2/M population by one day in both cell lines, albeit to a lesser degree (Figure 5). By five days, a subdiploid population indicative of apoptosis of $16.9 \%$ was evident in the HT-29 cells and $9.8 \%$ in the SW620 cells (Figures $6 A$ and $B$ ). The p53-expressing cells accumulated in G1 phase by one day following infection in the HT-29 cells but little alteration occurred in SW620 cells. Five days post Adp53 infection, approximately $5 \%$ were now subdiploid in both cell lines. The co-infection of Ad-E2F-1 and Ad-p53 


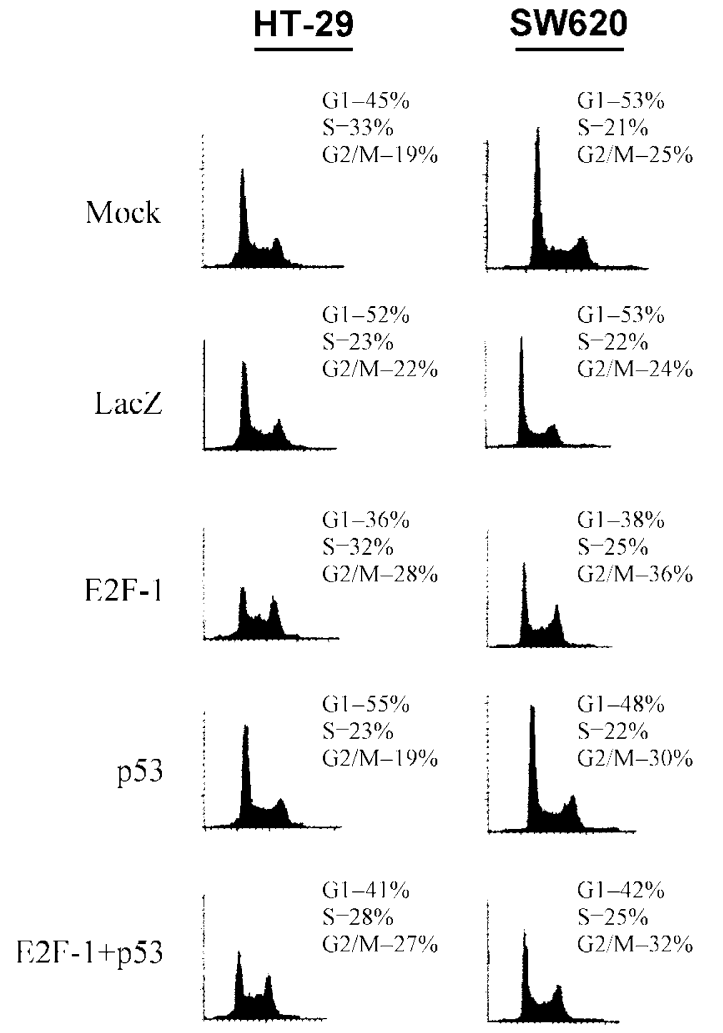

Figure 5. FACS cell cycle analysis. The flow cytometry profiles are shown here for the HT-29 and SW620 cell lines 1 day following infection. Histograms are representative of three separate experiments and percentages are the mean of three separate experiments. Ad-E2F-1 infection alone results in an increase in G2/M phases in both cell lines, whereas p53 induces a G1 accumulation in HT-29 cells but does not alter SW620 cells to any significant degree at the one day time point. Coexpression of p53 with E2F-1 inhibits E2F-1-induced G2/M accumulation in both cell lines. Remainder of cells is found in subG1 populations.

was associated with fewer cells accumulating in G2/M phase and fewer apoptotic (sub-G1) cells in HT-29's (11.9\%) and SW620's (7.3\%) as compared to E2F-1expressing cells only. Mock-infected and Ad-LacZinfected cells showed little alteration of normal cell cycle profiles and few subdiploid cells. These data suggest that the coexpression of p53 inhibits E2F-1-induced G2/ $M$ accumulation and DNA fragmentation.

\section{Confirmation of apoptosis}

Cells were examined for morphological changes consistent with apoptosis including cytoplasmic blebbing, chromatin condensation, nuclear fragmentation, and the appearance of apoptotic bodies. Cells infected with AdE2F-1 or Ad-p53 alone or in combination induced morphological changes indicative of apoptosis within five days (data not shown). Such changes were not apparent in mock-infected or Ad-LacZ-infected controls. To estimate the percentage of apoptotic cells in each sample, one thousand cells were counted and scored
A

H-29

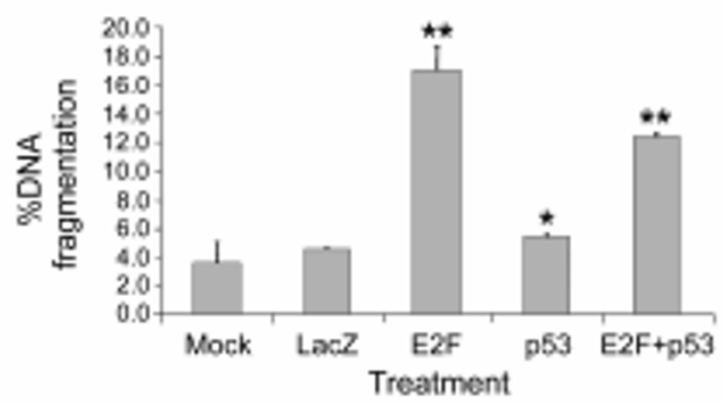

B

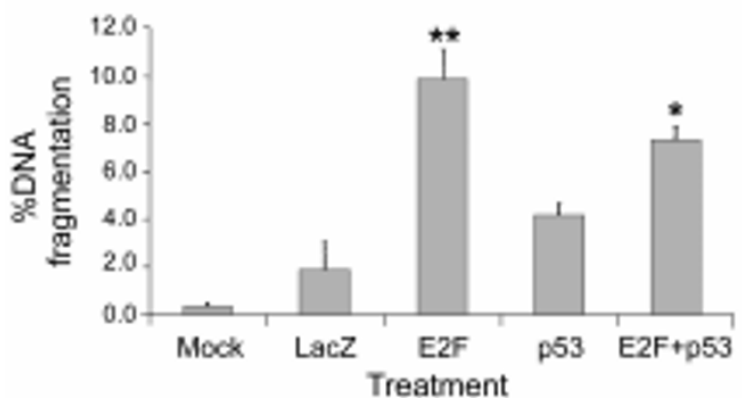

Figure 6. Effect of adenoviral gene transfer on DNA fragmentation (sub-G1 population) indicative of apoptosis. HT-29 and SW620 cells were treated by mock infection, infection with Ad-LacZ, Ad-E2F-1 or Ad-p53 alone or in combination. Samples were collected at the 5 day time point, DNA was stained with Propidium lodide and DNA fragmentation was measured by flow cytometry. Each data point represents the mean \pm SE of three separate experiments. $P$-values vs Ad-LacZ control are ${ }^{*} \leq 0.05$ and ${ }^{* *} \leq .0 .005$.

as either normal, or apoptotic (condensed, intense chromatin staining and nuclear fragmentation). As shown in Figure 7, the percentage of apoptotic morphology in cells co-expressing E2F-1 and p53 was less than that seen in cells expressing only E2F-1. On the bases of these findings, co-expression of p53 with E2F-1 significantly inhibits E2F-1-mediated apoptosis in HT-29 and SW620 colon cancer cells.

To provide additional evidence consistent with apoptosis, we probed for cleavage of the nuclear enzyme PARP. PARP cleavage is thought to be one of the earliest events in the execution phase of apoptosis (Kaufmann et al., 1993; Lazebnik et al., 1994; Tewari et al., 1995). Although down regulation of full-length PARP was evident in the E2F-1-overexpressing cells in both cell lines, we were unable to detect the $89 \mathrm{kD}$ PARP fragment which is most associated with apoptosis (Figure 8). In the SW620 cells overexpressing both E2F and p53, we also detected a decrease in full-length PARP $(113 \mathrm{kD})$ however, here again, we did not detect the $89 \mathrm{kD}$ apoptotic fragment. Dual gene transfer of p53 with E2F1 inhibited E2F-1-mediated downregulation of full-length PARP in the HT-29 cells but not in the SW620 cells. We have demonstrated previously that Ad-E2F-1 at $\mathrm{MOI}$ 

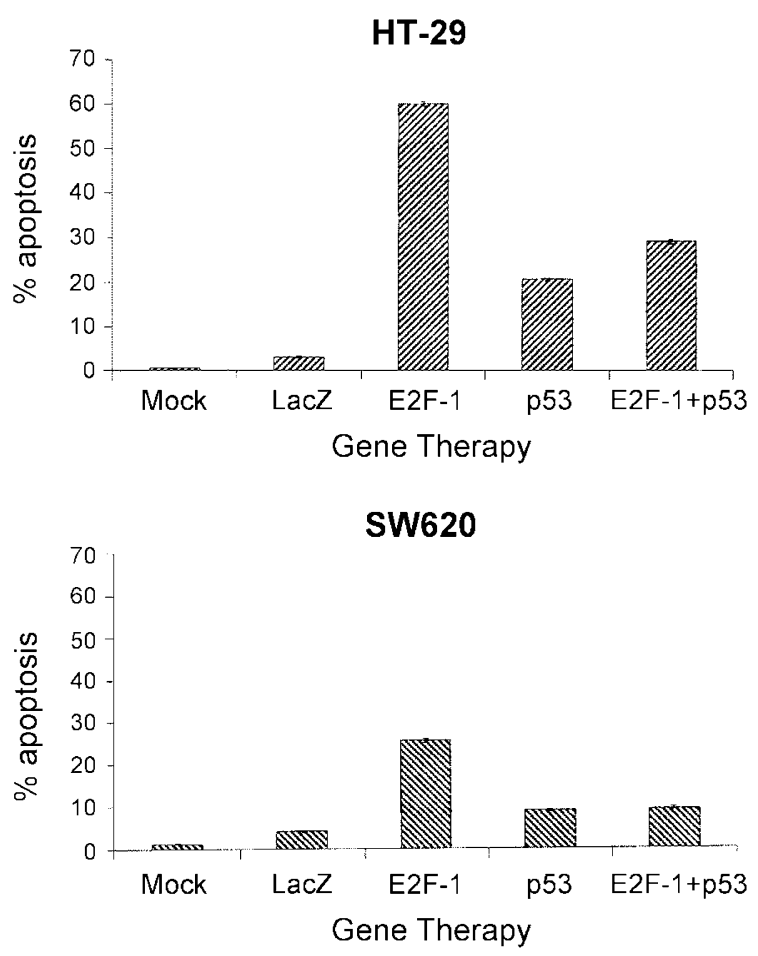

Figure 7. The proportion of morphologically apoptotic cells were estimated by examination of cell cytospins 5 days following mock infection and adenoviral gene transfer of E2F-1 or p53 alone or in combination. Each data point represents the mean $\pm \mathrm{SE}$ of two separate experiments. $P$-values for treatment vs Ad-LacZ control are <.01.

100 activates the caspase cascade and induces cleavage of PARP (Yong et al., Dong et al. and non-published data). Although we could not detect the $89 \mathrm{kD}$ PARP fragment following infection with the reduced MOI of AdE2F-1 used in these experiments, apoptosis is believed to be the mode of death based on the apoptotic morphology observed.

\section{Upregulation of p21 WAF1/CIP1}

The cyclin-dependent kinase inhibitor, p21 $1^{\text {WAF } 1 / C I P 1}$, is a transcriptional target of $\mathrm{p53}$ and is known to cause cells to arrest in both $\mathrm{G} 1$ and G2/M phases of the cell cycle. Recently, E2F-1 has also been shown to upregulate p21 and subsequently, E2F-1 activity is inhibited, thus forming a negative feedback loop (Hiyama et al., 1998). To further investigate the mechanisms of E2F-1 and p53 interactions, we probed for p21. We found that cells infected with Ad-E2F-1, Ad-p53 and Ad-E2F-1/Ad-p53 all exhibited increased expression of p21 in the HT-29 cell line, but only those SW620 cells infected with Adp53 either alone or in combination with Ad-E2F-1 showed upregulation of p21 (Figure 7). Interestingly, in the HT-29 cells, although p21 was upregulated to a similar degree in the E2F-1-expressing cells, only those cells co-expressing wild-type p53 were able to inhibit E2F-1-

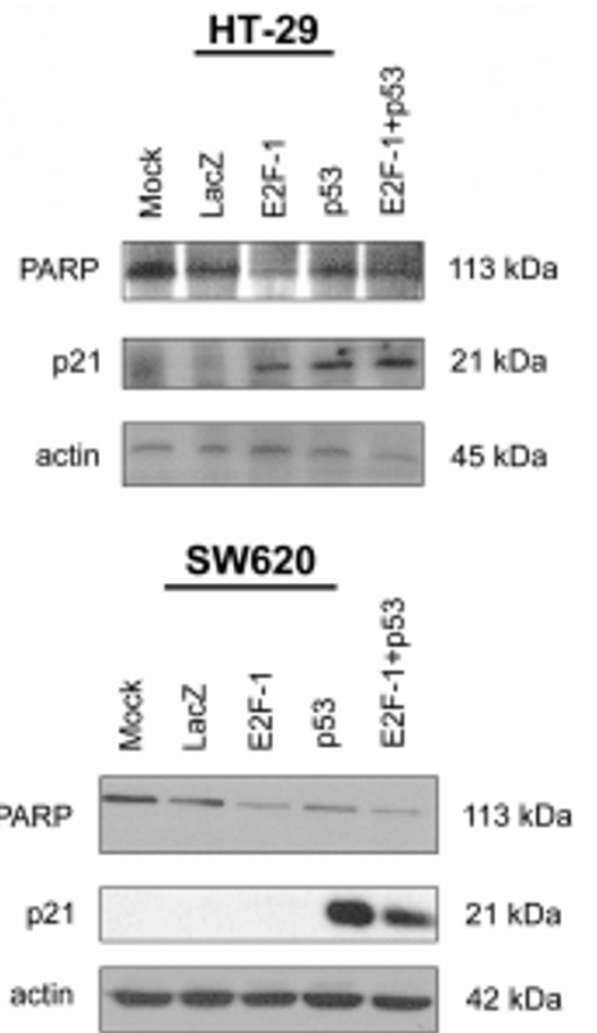

Figure 8. PARP and p21 expression at 5 days following treatment. Fulllength (113 kD) PARP is down regulated in E2F-1-expressing cells in both cell lines. p53 inhibits E2F-1-mediated downregulation of PARP in HT-29 cells but not in SW620 cells. p21 upregulated following overexpression of E2F-1 and p53 in HT-29 cells but SW620 cells demonstrated an increase in p21 protein only in the WT p53-overexpressing cells. Actin is shown to demonstrate similar levels of protein loading for each of the samples.

mediated G2/M accumulation and apoptosis (compare Figures 6 and 7). These data indicate that p53 may cooperate with p21 to inhibit E2F-1 activity. Additionally, although Ad-p53 induced p21 in SW620's, a G1 arrest was not achieved and may be due to other inherent mutations in this cell line.

\section{Discussion}

Considerable debate surrounds the role of p53 in the E2F-1-mediated apoptotic pathway. Early studies of E2F-1-mediated apoptosis suggest that the cooperation of wild-type p53 is necessary to induce cell death (Qin et al., 1994; Wu and Levine, 1994; Kowalik et al., 1995; Sladek, 1996; Phillips et al., 1997; Hsieh et al., 1997; Kowalik et al., 1998), while subsequent studies report that E2F-1-mediated apoptosis can occur through a p53-independent pathway (Berry et al., 1996; Hunt et al., 1997; Dong et al., 1999; Yang et al., 1999; Phillips et al., 1999). Combination E2F-1 and p53 gene therapy in a variety of tumor cell lines confirms the dual ability of 
E2F-1 to induce apoptosis through p53-dependent and -independent pathways (Agah et al., 1997; Frank et al., 1998; Bates and Vousden, 1999). Taken together, these data imply that cell lineage and genetic alterations of particular tumor cells play an important role in whether or not p53 is required in E2F-1-mediated apoptosis.

The mechanism of E2F-1-induced apoptosis remains ambiguous. It has been suggested that apoptosis and cell cycle progression are separable functions of E2F-1 (Phillips et al., 1997). Although DNA binding is required, transactivation is not necessary for the induction of apoptosis by E2F-1 (Hsieh et al., 1997). It is believed that E2F-1 induces a p53-dependent apoptotic response by an accumulation of p53 (Hiebert et al., 1995; Kowalik et al., 1998) that involves the induction of human $\mathrm{p} 14^{\mathrm{ARF}}$, which can then stabilize p53 levels by blocking MDM2mediated degradation of p53 (Haupt et al., 1997; Kubbutat et al., 1997; Bates et al., 1998). One p53independent pathway has been proposed that suggests E2F-1 potentiates cell death by blocking anti-apoptotic signaling pathways thereby sensitizing cells to apoptosis by a number of factors other than p53 (Phillips et al., 1999).

The mechanism by which p53 induces cell cycle arrest and apoptosis are better understood. In response to DNA damage, the p53 protein is stabilized by phosphorylation and transactivates genes that are associated with cell-cycle arrest (p21 ${ }^{\text {WAF1/CIP1 }}$ ) or death (Bax) (Miyashita et al., 1994; Ko and Prives, 1996; Cox, 1997; Sherr and Roberts, 1999). Variation in p53 induction is thought to play a key role in the choice between cellcycle arrest and death (Chen et al., 1996). Under conditions of modest genomic damage, the detection of lower levels of p53 correlates with p21 induction and cell-cycle arrest to halt cell growth and allow the initiation of DNA repair (Bates and Vousden, 1999). Whereas, more extensive DNA damage induces higher levels of p53, Bax induction and initiation of the apoptotic cascade (Bates and Vousden, 1999).

We have shown previously that infection with Ad-E2F1 at an MOI of 100 triggers swift apoptosis in HT-29 and SW620 cells (unpublished data). For purposes of these studies, we used reduced levels of Ad-E2F-1 (MOI 20) in order to detect cooperative or inhibitory effects in the E2F-1/p53 combination therapy. HT-29 and SW620 cells are colon adenocarcinomas with mutant p53 genes; when infected with Ad-E2F-1 at $\mathrm{MOI} 20$, these cells displayed a reduction in cell growth and viability (Figures 3 , 4). Cell cycle analysis revealed an E2F-1-induced accumulation of cells in G2/M phase by $24 \mathrm{~h}$ (Figure 5) and a subsequent significant subdiploid population of cells by 5 days in both cell lines (Figure 6 A \& B). Although we did not detect cleavage of PARP to the apoptotic $89 \mathrm{kD}$ fragment, Wright/Giemsa staining displayed a significant number of cells with condensed morphology, intense chromatin staining and nuclear frag- mentation consistent with death by apoptosis (Figure 7). Therefore, we found that even at the reduced levels used in these experiments, cells treated with Ad-E2F-1 still exhibited apoptosis as the mode of death. Furthermore, E2F-1 mediated apoptosis in these colon cancer cells is not dependent on the presence of wild-type p53.

Though E2F-1 mediated apoptosis is not p53 dependent in our in vitro colon cancer cell models, the effects of co-expressing wild-type p53 with E2F-1 transgene expression have not been clearly defined. It has been postulated that the re-introduction of wild-type p53 into transformed cells may sensitize cycling cells to chemotherapy by altering cell cycle dynamics and driving cells to a G1 cell cycle arrest or apoptosis (Clayman et al., 1993; Liu et al., 1994; Liu et al., 1995). Alternatively, wild-type p53 may assume functionality by binding with E2F-1 and activating alternative proapoptotic mechanisms (Wu and Levine, 1994; Qin et al., 1994; Sladek, 1996; Frank et al., 1998; Kowalik et al., 1998). Interestingly, Viashnav and Pant have reported that p53 specifically inhibits activated transcription of certain members of E2F family; however such inhibition was shown not to occur with E2F-1 (Vaishnav and Pant, 1999), supporting our hypothesis that E2F-1 and p53 might complement each other in the pathway to apoptosis. However, in our colon cancer cells, the simultaneous overexpression of the p53 transgene with the E2F1 transgene was shown to antagonize E2F-1 mediated apoptosis. In fact, concomitant p53 expression counteracted E2F-1-mediated G2/M accumulations and reproducibly reduced its apoptotic effect. Additionally, the cells co-infected with Ad-E2F-1 and Ad-p53 demonstrated cell viability that was intermediate between the levels of cell viability associated with E2F-1 or p53 gene transfer alone (Figure 4). Morphological evidence of apoptosis in the co-infected cells was markedly less than that seen in cells infected only with Ad-E2F-1 (Figure 7). Furthermore, simultaneous infection of AdE2F-1 with Ad-p53 inhibited the expression of virallyexpressed E2F-1 (Figure 2). We assume, this diminished expression of the E2F-1 protein was responsible, at least in part, for the reduced apoptosis seen in cells co-infected with Ad-E2F-1 and Ad-p53. Although we found no evidence of a cooperative effect at any dose levels tested, we cannot rule out the possibility that some combination of Ad-E2F-1 or Ad-p53 might produce a cooperative effect.

Interestingly, cells expressing the E2F-1 transgene, the p53 transgene, or both induced the expression of the CDK inhibitory protein, p2 $1^{\text {WAF } 1 / C I P 1}$ in the HT-29 cells (Figure 8 ). It is well documented that factors other than p53 can induce p21 gene expression (Li et al., 1995; Halevy et al., 1995; Qu et al., 1996; Greenhalgh et al., 1996; Billion et al., 1996; Lee et al., 1998). Our data suggest that E2F-1 may have a similar ability to induce p21 under certain circumstances and is in 
agreement with a recent study where E2F-1 was found to transactivate p21 (Hiyama et al., 1998). Although one study reported that $\mathrm{p} 21$ growth arrest fails to protect gliomal cells from E2F-1-mediated apoptosis (GomezManzano et al., 1999), a growing body of evidence now points to an opposing relationship between E2F-1 and p21 in both cell cycle control and induction of apoptosis (Chen et al., 1995; Shiyanov et al., 1996; Dimri et al., 1996; Hiyama et al., 1998; Delavaine and La Thangue, 1999). In the HT-29 cells, E2F-1-induced p21 did not prevent E2F-1-mediated apoptosis when expressed in the absence of wild-type p53. However, when p21 was expressed in the presence of wild-type p53, cell cycle analysis revealed a reduction in E2F-1-mediated $S$ phase entry, G2/M accumulation and cell death by apoptosis. Therefore, these findings suggest that in the presence of wild-type p53, p21 acts as an antagonist to E2F-1-mediated apoptotic pathways.

One possible explanation for the inability of p21 to effectively inhibit E2F-1-mediated apoptosis in the absence of wild-type p53 is that the level of p21 seen in the Ad-E2F-1-infected cells may not be sufficient to prevent the activity of massively overexpressed E2F-1 mediating cell cycle progression. Co-expression of p53 with E2F-1 may provide additional cellular interactions that enhance the ability of p21 to successfully halt the cell cycle at the G1 checkpoint, rendering the cell resistant to E2F-1-associated G2/M accumulation and apoptosis.

We have demonstrated that adenovirus-mediated E2F1 gene transfer is able to induce apoptosis in mutant p53 colon cancer cells. Combination E2F-1 and p53 gene transfer did not offer a therapeutic advantage in colon cancer cells in vitro; rather, the combined adenovirus-mediated wild type p53/E2F-1 gene transfer inhibited E2F-1 expression, E2F-1-mediated growth inhibition and E2F-1-induced apoptosis. We have also shown that the overexpression of E2F-1 was able to induce the expression of endogenous p21 in the HT-29 cell line but not in SW620 cells. Unlike the well-described induction of p21 by wild-type p53, E2F-1 mediated induction of p21 did not result in a G1 cell cycle arrest. Instead, E2F1 mediated induction of p21 was associated with accumulation of cells in G2/M and cellular commitment to apoptosis. Further study into the role of p21 may provide a possible link to the actions of p53 and E2F-1 and their complex interplay in apoptosis and the regulation of the cell cycle.

\section{Acknowledgements}

We are grateful to Dr. Brent French for providing the Ad5CMV-LacZ, and Dr. T.J. Liu for providing the Ad5CMV-E2F-1, and Dr. Bert Vogelstein for providing the p53 adenovirus. We thank Sherri Matthews for expert assistance with manuscript preparation. Support- ed by Grant 96-55 from the American Cancer Society, Grant 96-46 from the Alliant Community Trust Fund, The Mary and Mason Rudd Foundation Award, and the Center for Advanced Surgical Technologies of Norton Hospital.

\section{References}

Agah R, Kirshenbaum LA, Abdellatif M, Truong LD, Chakraborty S, Michel LH, Schneider MD. Adenoviral delivery of E2F-1 directs cell cycle reentry and $\mathrm{p}-53$ independent apoptosis in postmitotic adult myocardium in vivo. J Clin Invest 1997;100:2722-28

Bates S, Phillips AC, Clark PA, Scott F, Peters G, Ludwig RL, Vousden $\mathrm{KH}$. p14 links the tumor suppressors Rb and p53. Nature 1998;395:125-25

Bates S, Vousden $\mathrm{KH}$. Mechanisms of p53-mediated apoptosis. Cell Mol Life Sci 1999;55:28-37

Billion N, van Grunsven LA, Rudkin BB. The CDK inhibitor

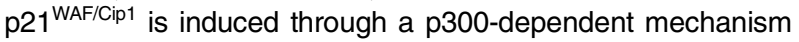
during NGF-mediated neuronal differentiation of PC12 cells. Oncogene 1996;13:2047-54

Chen J, Jackson PK, Kirschner MW, Dutta A. Separate domains of p21 involved in the inhibition of Cdk kinase and PCNA. Nature 1995;374:386-88

Chen X, Ko LJ, Jayaraman L, Prives C. p53 levels, functional domains and DNA damage determine the event of apoptotic response of tumor cells. Gened Dev 1996;10:2438-51

Clayman GL, El-Naggar AK, Roth JA, Zhang WW, Goepfert $\mathrm{H}$, Taylor DL, Liu TJ. In vivo molecular therapy with p53 adenovirus for microscopic residual head and neck squamous carcinoma. Cancer Res 1993;53:4129-33

Cox LS. Multiple pathways control cell growth and transformation: overlapping and independent activities of p53

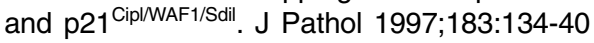

Delavaine L, La Thangue NB. Control of E2F activity by p21 ${ }^{\text {Wafl/Cipl. }}$ Oncogene 1999;18:5381-92

Dimri GP, Nakanishi M, Desprez PY, Smith JR, Campisis J. Inhibition of E2F activity by the cyclin-dependent protein kinase inhibitor p21 in cells expressing or lacking a functional retinoblastoma protein. Mol Cell Biol 1996;16:2987-97

Dong YB, Yang HL, Elliott MJ, Liu TJ, Stilwell A, Atienza C Jr, McMasters KM. Adenovirus-mediated E2F-1 gene transfer efficiently induces apoptosis in melanoma cells. Cancer 1999;86:2021-33

Dyson N. The regulation of E2F by pRB-family proteins. Genes Dev 1998;12:2245-62

El-Deiry WS, Tokino T, Velculescu VE, Levy DB, Parson R, Trent JM, Lin D, Mercer WE, Kinzler KW, Vogelstein B. WAF1, a potential mediator of p53 tumor suppression. Cell 1993;75: 817-25

Frank DK, Liu TJ, Frederick MJ, Clayman GL. Combination E2F-1 and p53 gene transfer does not enhance growth 
inhibition in human squamous cell carcinoma of the head and neck. Clin Cancer Res 1998;4:2265-72

Fueyo J, Gomez-Manzano C, Yung WKA, Liu TJ, Alemany R, McDonnel TJ, Shi X, Roa JS, Levin VA, Kyritsis AP. Overexpression of E2F-1 in glioma triggers apoptosis and suppresses tumor growth in vitro and in vivo. Nat Med 1998;4:685-89

Goldsmith ME, Gudas Jm, Schneider E, Cowan KH. Wild type p53 stimulates expression from the human multidrug resistance in a p53-negative cell line. J Biol Chem 1995; 270(4):1894-98

Gomez-Manzano C, Fueyo J, Alameda F, Kyritsis AP, Yung WK. Gene therapy for gliomas: p53 and E2F-1 proteins and the target of apoptosis. Int J Mol Med 1999;3:81-85

Greenhalgh DA, Wang XJ, Donehower LA, Roop DR. Paradoxical tumor inhibitory effect of p53 loss in trangenic mice expressing epidermal-targeted vHa-ras, v-fos, or human transforming growth factor alpha. Cancer Res 1996;56:441323

Halevy O, Novitch BG, Spicer DB, Skapek SX, Rhee J, Hannon GJ, Beach D, Lassar AB. Correlation of terminal cell cycle arrest of skeletal muscle with induction of p21 by MyoD. Science 1995;267:1018-21

Harper JW, Adami GR, Wei N, Keyomarsi K, Elledge SJ. The p21 cdk-interacting protein Cip1 is a potent inhibitor of G1 cyclin-dependent kinases. Cell 1993;75:805-16

Haupt Y, Maya R, Kazaz A, Oren M. Mdm2 promotes the rapid degradation of p53. Nature 1997;387:296-99

Helin K. Regulation of cell proliferation by the E2F transcription factors. Curr Opin Genet Dev 1998;8:28-35

Hiebert SW, Packham G, Strom DK, Haffner R, Oren M, Zambetti G, Cleveland JL. E2F-1: DP-1 induces and overrides survival factors to trigger apoptosis. Mol Cell Biol 1995;15: 6864-74

Hiyama $\mathrm{H}$, lavarone $\mathrm{A}$, Reeves $\mathrm{S}$. Regulation of the cdk inhibitor p21 gene during cell cycle progression is under the control of the transcrpition factor E2F. Oncogene 1998;16: 1513-23.

Hsieh JK, Frederdorf S, Kouzarides T, Martin K, Lu X. E2F1induced apoptosis requires DNA binding but not transactivation and is inhibited by the retinoblastoma protein through direct interaction. Genes Dev 1997;11:1840-52

Hunt K, Deng J, Liu T, Wilson-Heiner M, Swisher SG, Clayman G, Hung M. Adenovirus-mediated overexpression of the transcription factor E2F-1 induces apoptosis in human breast and ovarian carcinoma cell lines and does not require p53. Cancer Res 1997;57:4722-26

Johnson DG, Scwarz JK, Cress WD, Nevins JR. Expression of transcription factor E2F1 induces quiescent cells to enter $S$ phase. Nature 1993;365:349-52

Kastan MB, Onyekwere O, Didransky D, Vogelstein B, Craig RW. Participation of p53 protein in the cellular response to DNA damage. Cancer Res 1991;51:6304-11

Kaufman SH, Desnoyers S, Ottaviano Y, Davidson NE, Poirer
GG. Specific protolytic cleavage of poly(ADP-ribose) polymerase: an early marker of chemotherapy-induced apoptosis. Cancer Res 1993;53:3976

Kernohan NM, Cox LS. Regulation of apoptosis by Bcl-2 and its related proteins: immunochemical challenges and therapeutic implications. J Pathol 1996;179:1-3

Ko LJ, Prives C. p53: puzzle and paradigm. Genes Dev 1996;10:1054-72

Kowalik TF, DeGregori J, Jakoi L, Nevins JR. E2F1-specific induction of apoptosis and p53 accumulation, which is blocked by Mdm2. Cell Growth Differ 1998;9:113-18

Kowalik TF, DeGrgori J, Schwarz JK, Nevins JR. E2F1 overexpression in quiescent fibroblasts leads to induction of cellular DNA synthesis and apoptosis. J Virol 1995;69:2491500

Kubbutat $\mathrm{MH}$, Jones SP, Vousden $\mathrm{KH}$. Regulation of p53 stability by Mdm2. Nature 1997;387:299-303

Lazenbnik YA, Kaufmann SH, Desnoyers S, Poirier GG, Earnshaw WC. Cleavage of poly(ADP-ribose) polymerase by a proteinase with properties like ICE. Nature 1994;371:346

Lee SJ, Ha MJ, Lee J, Nguyen P, Choi YH, Pirnia F, Kang WK, Wang XF, Kim SJ, Trepel JB. Inhibition of the 3-hydroxy3-methylglutaryl-coenzyme A reductase pathway induces p53-independent transcriptional regulation of $\mathrm{p} 21^{\text {WAF1/Cip } 1}$ in human prostate carcinoma cells. J Biol Chem 1998;273: 10618-23

Li CY, Suardet L, Little JB. Potential role of WAF1/Cipl/p21 as a mediator of TGF-beta cytoinhibitory effect. J Biol Chem 1995;270:4971-74

Liu TJ, El-Naggar AK, McDonnell TJ, Steck KD, Wang M, Taylor DL, Clayman GL. Apoptosis induction mediated by wild-type p53 adenoviral gene transfer in squamous cell carcinoma of the head and neck. Cancer Res 1995;55:311722

Liu TJ, Wang M, Breau RL, Henderson Y, El-Nagar AK, Steck $\mathrm{KD}$, Sicard MW, Clayman G. Apoptosis induction by E2F-1 via adenoviral-mediated gene transfer results in growth suppression of head and neck squamous cell carcinoma cell lines. Cancer Gene Ther 1999;6:163-71

Liu TJ, Zhang WW, Taylor DL, Roth JA, Geopfert H, Clayman GL. Growth suppression of human head and neck cancer cells by the introduction of wild-type p53 gene via a recombinant adenovirus. Cancer Res 1994;54:3662-67

Meng RD, Phillips P, El-Diery WS. p53-independent increase in E2F-1 expression enhances the cytotoxic effects of etoposide and of adriamycin. Int J Oncol 1999;14:5-14

Merchant AK, Loney TL, Maybaum J. Expression of wild-type p53 stimulates an increase in both $\mathrm{Bax}$ and $\mathrm{Bcl}-\mathrm{xl}$ protein content in HT29 cells. Oncogene 1996;13:2631-37

Miyahita T, Harigai M, Reed JC. Identification of a p53dependent negative response element in the bcl-2 gene. Cancer Res 1994;54:3131-35

Miyashita T, Reed JC. Tumor suppressor p53 is a direct transcriptional activator of the human bax gene. Cell 1995;80: 
293-99

Nelson WG, Kastan MB. DNA strand breaks: the DNA template alterations that trigger p53-dependent DNA damage response pathways. Mol Cell Biol 1994;14:1815-23

Phillips AC, Ernst MK, Bates S, Rice NR, Vousden KH. E2F1 potentiates cell death by blocking antiapoptotic signaling pathways. Mol Cell 1999;4:771-81

Phillips AC, Bates S, Ryan KM, Helin K, Vousden KH. Induction of DNA synthesis and apoptosis are separable functions of E2F-1. Genes Dev 1997;11:1853-63

Qin XQ, Livingston DM, Kaelin WG Jr, Adams PD. Deregulated transcription factor E2F-1 expression leads to Sphase entry and p53-mediated apoptosis. Proc Natl Acad Sci USA 1994;91:10918-22

Qiu X, Forman HJ, Schonthal AH, Cadenas E. Induction of p21 mediated by reactive oxygen species formed during the metabolism of aziridinylbenzoquinones by HCT116 cells. J Biol Chem 1996;271:31915-21

Sherr CJ, Roberts JM. CDK inhibitors: positive and negative regulators of $\mathrm{G}_{1}$-phase progression. Genes Dev 1999;13: 1501-12
Shiyanov P, Bagchi S, Adami G, Kokontis J, Hay N, Arroyo M, Morozov A, Raychaudhuri P. p21 disrupts the interaction between cdk2 and the E2F-p130 complex. Mol Cell Biol 1996; $16: 737-44$

Sladek TL. Established cell lines transformed by E2F-1 overexpression contain wild-type p53. Cell Prolif 1996;29: $579-88$

Tewari M, Quan LT, O'Rourke K, Desnoyers S, Zeng Z, Biedler DR, Poirer GG. Salvesen that cleaves the death substrate poly(ADP-ribose) polymerase. Cell 1995;81:801-9

Vaishnav $Y N$, Pant $V$. Differential regulation of E2F transcription factors by p53 tumor suppressor protein. DNA Cell Biol 1999;18:911-22

Wu XW, Levine AJ. p53 and E2F-1 cooperate to mediate apoptosis. Proc Natl Acad Sci USA 1994;91:602-606

Yang HL, Dong YB, Elliott MJ, Liu TJ, Atienza C Jr, Stillwell A, McMasters KM. Adenovirus-mediated E2F-1 gene transfer inhibits MDM2 expression and efficiently induces apoptosis in MDM2-overexpressing tumor cells. Clin Cancer Res 1999;5: 2242-50 